\title{
PROSPECTIVE RANDOMIZED SUBJECT- MASKED STUDY OF INTRAVITREAL BEVACIZUMAB MONOTHERAPY VERSUS DEXAMETHASONE IMPLANT MONOTHERAPY IN THE TREATMENT OF PERSISTENT DIABETIC MACULAR EDEMA
}

\author{
SANKET U. SHAH, MD, * ASHLEY HARLESS, CRC,$\dagger$ LAURA BLEAU, LPN, $\dagger$ RAJ K. MATURI, MD*†
}

\begin{abstract}
Purpose: To compare intravitreal bevacizumab monotherapy with intravitreal dexamethasone delayed delivery system monotherapy for persistent diabetic macular edema.

Methods: Single-center, randomized, subject-masked study of eyes with persistent diabetic macular edema, defined as central subfield thickness (CST) $>340 \mu \mathrm{m}$ despite $\geq 3$ anti-vascular endothelial growth factors injections within 5 months. The intravitreal bevacizumab monotherapy ( $n=23$ eyes) and delayed delivery system monotherapy $(n=27$ eyes) groups received treatments q1month and q3months, respectively.
\end{abstract}

Results: Baseline best-corrected visual acuity and CST were similar in the two groups. At Month 7, the mean final best-corrected visual acuity (mean \pm SD) was $65 \pm 16$ letters (mean Snellen visual acuity 20/50) and $64 \pm 11$ letters $(20 / 50)(P=0.619)$, the mean change in best-corrected visual acuity was $+5.6 \pm 6.1$ and $+5.8 \pm 7.6$ letters $(P=0.785)$, the mean final CST was $471 \pm 157$ and $336 \pm 89 \mu \mathrm{m}(P=0.001)$, and the mean change in CST was $-13 \pm 105$ and $-122 \pm 120 \mu \mathrm{m}(P=0.005)$ in the intravitreal bevacizumab monotherapy and delayed delivery system monotherapy groups, respectively. The number of injections was $7.0 \pm 0.2$ and $2.7 \pm 0.5(P<0.001)$ in the 2 groups.

Conclusion: The two groups had similar best-corrected visual acuity gains. The delayed delivery system monotherapy group achieved a significantly greater reduction of CST compared with the intravitreal bevacizumab monotherapy group, with a q3month interval of treatment, and had no recurrent edema at any visit.

RETINA 36:1986-1996, 2016

$\mathrm{D}$ iabetes is the most common cause of new blindness in the working-age adults aged 20 years to 74 years in the United States. ${ }^{1}$ Approximately 1 in every 25 diabetic patients aged 40 years or older has diabetic macular edema (DME) in at least one eye in the United States. ${ }^{2}$

Anti-vascular endothelial growth factor (antiVEGF) agents have become the new gold standard for the treatment of DME after several randomized controlled trials demonstrated their superiority over laser photocoagulation. ${ }^{3-6}$ However, the DME control with anti-VEGF agents is not without exception, and, in fact, a significant proportion of eyes continue to have persistent DME despite treatment.

In DRCR protocol I, $40 \%$ of patients in the 2 ranibizumab groups had residual macular edema with central subfield thickness (CST) exceeding $250 \mu \mathrm{m}$ after 2 years despite strict adherence to study protocol. ${ }^{4}$ The RISE/RIDE combined data showed that in ranibizumab-treated eyes, at least $26 \%$ have continued edema and approximately $74 \%$ of eyes have angiographic leakage at 2 years. ${ }^{5}$ In DRCR Protocol $\mathrm{T}$, CST remained greater than $250 \mu \mathrm{m}$ in $44 \%$ of aflibercept-treated eyes, $64 \%$ of bevacizumabtreated eyes, and $42 \%$ of ranibizumab-treated eyes at 1 year. ${ }^{7}$ There is indirect evidence that such persistent macular edema can cause permanent vision loss, possibly related to prolonged disruption of retinal architecture from the edema, producing irreversible retinal damage. In the 36-month results of RISE/ RIDE, patients who received monthly $0.5 \mathrm{mg}$ 
ranibizumab after an initial 2 years of sham treatment failed to achieve the same degree of visual improvement as those patients who were treated continuously with monthly $0.5 \mathrm{mg}$ ranibizumab, despite having a similar reduction in macular edema. ${ }^{8}$

Although no randomized studies were found that evaluated patients with DME unresponsive to bevacizumab, several nonrandomized prospective studies have evaluated this question. The REEF study attempted to switch from one anti-VEGF agent to another and showed ranibizumab to achieve visual and anatomical improvements in some of the eyes with DME resistant to bevacizumab therapy, but as expected, the control was not universal. ${ }^{9}$ The use of laser to supplement anti-VEGF therapies has been previously advocated and studied. However, recent studies, including the DRCR.net 3-year results and a 5-year multicenter randomized controlled trial, established the lack of discernable visual acuity benefit of the addition of laser to anti-VEGF agents over the long term for DME control. ${ }^{10,11}$ Therefore, there is a need for a different agent to treat this subset of eyes with persistent DME unresponsive to anti-VEGF agents.

The dexamethasone implant (Ozurdex, Allergan, Inc., Irvine, CA) was approved by the US Food and Drug Administration in September 2014 for the treatment of DME. Our previous 12-month study comparing bevacizumab with dexamethasone combination therapy versus bevacizumab monotherapy for diabetic eyes that were partially responsive to anti-VEGF treatment showed that macular edema reduction was significantly greater in the combination group versus the monotherapy group $(-45 \mu \mathrm{m}$ vs. $-30 \mu \mathrm{m}, P=0.03)$ and that the visual acuity gain was similar in both groups $(+5.4$ letters vs. +4.9 letters, $P=0.75) .{ }^{12}$ Also, we learned that the macular edema improvement did not last the

From the *Department of Ophthalmology, Indiana University School of Medicine, Indianapolis, Indiana; and $†$ Midwest Eye Institute, Indianapolis, Indiana.

Supported by an investigator-initiated trial grant from Allergan, Inc. Presented as an oral presentation at the American Society of Retina Specialists annual meeting in Vienna, Austria, July 14, 2015.

SUS, AH, and LB have no conflicts of interest. RKM acknowledges receiving research support from AbbVie, Allergan, Genentech, GlaxoSmithKline, KalVista, and Santen, and having consulting agreements with Allergan and Eli Lilly.

Supplemental digital content is available for this article. Direct URL citations appear in the printed text and are provided in the HTML and PDF versions of this article on the journal's Web site (www.retinajournal.com).

This is an open access article distributed under the terms of the Creative Commons Attribution-Noncommercial-Noderivatives License 4.0 (CC BY-NC-ND), which permits downloading and sharing the work provided it is properly cited. The work cannot be changed in any way or used commercially.

Reprint requests: Raj K. Maturi, MD, Midwest Eye Institute, Indianapolis, $200 \mathrm{~W}$ 103rd Street, Suite 1060, Indianapolis, IN 46290; e-mail: rmaturi@gmail.com full 4 months interval of treatment used in that study, and edema recurred to a significant extent after 3 months. ${ }^{12}$ Therefore, if dexamethasone implant alone, at q3month intervals, which seems to be the duration of clinical efficacy in patients with persistent DME, is found to be equivalent to current anti-VEGF agents such as bevacizumab (which is used q4weeksq6weeks) in treating persistent DME, then it would imply significantly fewer injections and possibly fewer patient visits. Therefore, in this randomized study, we directly compared bevacizumab monotherapy to dexamethasone monotherapy in the treatment of DME.

\section{Methods}

\section{Study Design}

This prospective, subject-masked, randomized, controlled trial was conducted at a single site. The institutional review board approved the study protocol and each subject provided written informed consent before enrollment. The study site complied with the Health Insurance Portability and Accountability Act. The study involved off-label use of intravitreal bevacizumab for the treatment of DME.

\section{Participants}

Eligible patients were recruited from the practice of the principal investigator. Included were patients with diabetes mellitus Type 1 or 2 , who were 18 years or older, with best-corrected visual acuity scores between 24 and 78 Early Treatment of Diabetic Retinopathy Study letters (20/32-20/320 Snellen equivalent), and with the presence of persistent DME which was defined as a CST of $>340$ $\mu \mathrm{m}$ measured by spectral domain optical coherence tomography (OCT) despite at least 3 anti-VEGF injections within the previous 5 months. Fluorescein angiography was performed at the baseline visit to rule out significant foveal nonperfusion. Previous treatment of DME was allowed, and most subjects had far more than the minimum that this study required. Exclusion criteria included any intravitreal anti-VEGF injection within the previous 4 weeks, intravitreal triamcinolone injection within the previous 8 weeks, intravitreal dexamethasone (Ozurdex) within the previous 16 weeks, and laser photocoagulation of the retina within the previous 16 weeks. Other exclusion criteria included active iris neovascularization, aphakia, pseudophakia with anterior chamber intraocular lens, significant epiretinal membrane or vitreomacular traction that could contribute to secondary macular edema, active or 
suspected ocular or periocular infections including most viral diseases of the cornea and conjunctiva including active epithelial keratitis due to herpes simplex, herpes zoster, vaccinia, mycobacteria, or fungi, a known history of intraocular pressure (IOP) increase because of corticosteroids that would not be adequately controlled with 2 topical glaucoma medications, hypersensitivity to any component of Avastin or Ozurdex, enrollment in an investigational drug study within the previous 30 days, and any condition that in the opinion of the investigator might compromise the results of the trial or preclude the patient from completing all study visits. Female patients of childbearing age were required to have a negative pregnancy test before enrollment and to use a reliable form of contraception throughout the study.

\section{Treatment Groups and Randomization}

After providing written consent, eligible subjects were randomly assigned to one of 2 treatments: 1) intravitreal bevacizumab (IVB) (Avastin, Genentech, Inc., South San Fancisco, CA) monotherapy or 2) intravitreal dexamethasone delayed delivery system implant (DDS) (Ozurdex) monotherapy (DDS) in a 1:1 ratio (see Figure, Supplemental Digital Content 1, http://links.lww.com/IAE/A439, which illustrates the study design). Randomization was achieved by nonstudy personnel using computer-generated allocations and with 2 sets of sealed envelopes (one set for baseline visual acuity $<65$ letters and another set for baseline visual acuity $\geq 65$ letters at baseline). For patients in whom both eyes met the eligibility criteria, the right eye was randomized to a treatment group and the left eye was assigned to the other group. The study duration was 7 months.

Eyes assigned to the IVB group received intravitreal injection of bevacizumab (Avastin) $1.25 \mathrm{mg}$ in $0.05 \mathrm{~mL}$ at baseline and monthly thereafter if retreatment criteria were met. If retreatment criteria were not met at any of the monthly visits, then sham injection was administered at those visits. Eyes assigned to the DDS group received intravitreal injection of the dexamethasone DDS implant (Ozurdex) $0.7 \mathrm{mg}$ at baseline, Month 3, and Month 6, and sham injections at Months 1, 2, 4, and 5. If retreatment criteria were not met at Month 3, then sham injection was administered at Month 3 and Ozurdex was then administered at Month 4. If retreatment criteria were not met at Month 6, then sham injection was administered at Month 6. Ozurdex could not be administered at an interval of less than 3 months.

Retreatment criteria included either visual acuity $<83$ letters or CST $\geq 300 \mu \mathrm{m}$. Retreatment was with- held only if visual acuity was $\geq 83$ letters and CST was $<300 \mu \mathrm{m}$. Escape criteria included an increase of CST by $\geq 200 \mu \mathrm{m}$ from baseline at any of the Months 2 through 6 for either group, which would qualify the subject for withdrawal from the study at the investigator's discretion.

\section{Outcomes and Statistical Analysis}

Outcomes were analyzed in terms of efficacy and safety. The primary efficacy measures included mean change in visual acuity from baseline to Month 7 and mean change in CST from baseline to Month 7. The secondary efficacy measures included mean number of 10- and 15-letter gainers from baseline to Month 7, mean time to 10-letter gain, mean change in visual acuity based on previous number of antiVEGF injections, and mean change in total macular volume from baseline to Month 7. The safety measures included glaucoma-related outcomes such as mean change in IOP, number of visits with IOP exceeding $21 \mathrm{mmHg}$, number of patients requiring addition of ocular antihypertensive medications or laser or incisional glaucoma surgery during the study, and mean change in OCT retinal nerve fiber layer average thickness. Other safety measures including cataracts, endophthalmitis, other ocular serious adverse events, and systemic serious adverse events were recorded as well.

The baseline characteristics of the cohort, including demographics, disease and treatment history, and functional and morphologic measurements, were summarized by descriptive statistics. The efficacy and safety measures were statistically analyzed using nonparametric tests, including Mann-Whitney $U$ test and Fisher's exact $t$-test, and using an intentto-treat approach.

\section{Results}

\section{Baseline Characteristics}

Between January 29, 2014, and October 15, 2014, 50 eyes of 45 patients (mean age $61 \pm 10$ years) were enrolled, with 23 and 27 eyes assigned to the IVB and DDS groups, respectively. The baseline characteristics of study subjects and enrolled eyes were similar in both groups as summarized in Table 1. In particular, average visual acuity, CST, and total macular volume were equivalent in both groups. The treatments that the eyes had received before study enrollment are summarized in Table 2, and were similar in both groups as well. 
Table 1. Bevacizumab Versus Dexamethasone Implant for Persistent Diabetic Macular Edema: Baseline Characteristics

\begin{tabular}{|c|c|c|c|}
\hline Feature & $\begin{array}{l}\text { IVB Group, Mean } \pm \text { SD } \\
(n=23 \text { Eyes })\end{array}$ & $\begin{array}{l}\text { DDS Group, Mean } \pm \text { SD } \\
(n=27 \text { Eyes })\end{array}$ & $P$ \\
\hline Age, yrs & $61 \pm 9$ & $65 \pm 11$ & 0.209 \\
\hline Gender, n (\%) & & & 0.571 \\
\hline Male & $13(57)$ & $12(44)$ & \\
\hline Female & $10(44)$ & $15(56)$ & \\
\hline Race, n (\%) & & & 0.614 \\
\hline White & $22(96)$ & $24(89)$ & \\
\hline African-American & 1 (4) & $3(11)$ & \\
\hline Diabetes mellitus, n (\%) & & & 0.090 \\
\hline Type 1 & $3(13)$ & $0(0)$ & \\
\hline Type 2 & $20(87)$ & $27(100)$ & \\
\hline Lens status, n (\%) & & & 0.407 \\
\hline Phakic & $14(61)$ & $13(48)$ & \\
\hline Pseudophakic & 9 (39) & $14(52)$ & \\
\hline $\begin{array}{l}\text { BCVA (ETDRS letters) } \\
\text { (mean Snellen visual acuity) }\end{array}$ & $59 \pm 13(20 / 63)$ & $59 \pm 12(20 / 63)$ & 0.770 \\
\hline $\mathrm{IOP}, \mathrm{mmHg}$ & $16 \pm 2$ & $15 \pm 2$ & 0.619 \\
\hline $\mathrm{CST}, \mu \mathrm{m}$ & $485 \pm 122$ & $458 \pm 100$ & 0.508 \\
\hline $\mathrm{TMV}, \mathrm{mm}^{3}$ & $12.0 \pm 1.9$ & $11.7 \pm 1.9$ & 0.350 \\
\hline
\end{tabular}

BCVA, best-corrected visual acuity; DDS, intravitreal dexamethasone delayed delivery system implant; ETDRS, early treatment diabetic retinopathy study; TMV, total macular volume.

\section{Follow-up}

Monthly evaluation, including the 7-month evaluation, was performed in $23(100 \%)$ eyes in the IVB group and 27 (100\%) eyes in the DDS group. None of the eyes were lost to follow-up in either group. Optical coherence tomography data were unavailable (or unable to be recorded) for two eyes (at one visit for one eye and at three visits for another eye) in the IVB group and for two eyes (at one visit for each of the two eyes) in the DDS group. Outcomes were calculated based on eyes with available data for each of the visits.

\section{Treatments}

Twenty-two (96\%) eyes in the IVB group met retreatment criteria at each of the scheduled injection visits, and received a total of 7 bevacizumab injections each. One (4\%) eye in the IVB group did not meet retreatment criteria at one of the visits, and received a total of 6 bevacizumab injections. Nineteen (71\%) eyes in the DDS group met retreatment criteria at each of the scheduled injection visits and received a total of 3 dexamethasone injections. Eight (29\%) eyes in the DDS group did not meet retreatment criteria at one of the scheduled visit, and received a total of 2 dexamethasone injections each. Number of injections per eye (mean $\pm \mathrm{SD})$ was $7.0 \pm 0.2$ in the IVB group and $2.7 \pm 0.5$ in the DDS group $(P<0.001)$ over 7 months. None of the eyes were withdrawn from the study at the discretion of the investigator.

\section{Efficacy Measures}

The visual outcomes in the 2 groups are summarized and compared in Table 3. As noted, all visual outcomes were similar in both groups (see Figure, Supplemental Digital Content 2, http://links.lww.com/ IAE/A440, which graphically illustrates the visual acuity over time). The change in visual acuity over time is graphically demonstrated in Figure 1. The number of

Table 2. Bevacizumab Versus Dexamethasone Implant for Persistent Diabetic Macular Edema: Summary of Treatments Before the Study

\begin{tabular}{|c|c|c|c|}
\hline Feature & $\begin{array}{l}\text { IVB Group, Mean } \pm \text { SD } \\
(n=23 \text { Eyes })\end{array}$ & $\begin{array}{l}\text { DDS Group, Mean } \pm \text { SD } \\
(n=27 \text { Eyes })\end{array}$ & $P$ \\
\hline Number of previous intravitreal anti-VEGF injections & $15 \pm 11$ & $18 \pm 12$ & 0.311 \\
\hline Interval from last intravitreal anti-VEGF injection, wk & $6 \pm 6$ & $6 \pm 4$ & 0.825 \\
\hline Number of previous intravitreal triamcinolone injections & $8 \pm 6(n=11)$ & $9 \pm 6(n=29)$ & 0.767 \\
\hline Interval from last intravitreal triamcinolone injection, wk & $41 \pm 19(n=11)$ & $89 \pm 111(n=29)$ & 0.22 \\
\hline Number of previous intravitreal DDS injections & $2 \pm 1(n=3)$ & $2 \pm 1(n=3)$ & 0.36 \\
\hline Interval from last intravitreal DDS injection, wk & $85 \pm 46(n=3)$ & $81 \pm 8(n=3)$ & 0.513 \\
\hline
\end{tabular}

DDS, intravitreal dexamethasone delayed delivery system implant; IVB, intravitreal bevacizumab. 
Table 3. Bevacizumab Versus Dexamethasone Implant for Persistent Diabetic Macular Edema: Visual Acuity Outcomes at Month 7

\begin{tabular}{|c|c|c|c|}
\hline Feature & $\begin{array}{l}\text { IVB Group, Mean } \pm \text { SD, } \\
\quad(n=23 \text { Eyes })\end{array}$ & $\begin{array}{l}\text { DDS Group, Mean } \pm \text { SD } \\
(n=27 \text { Eyes })\end{array}$ & $P$ \\
\hline Final BCVA (ETDRS letters) (mean Snellen visual acuity) & $65 \pm 16(20 / 50)$ & $64 \pm 11(20 / 50)$ & 0.619 \\
\hline Change in BCVA (ETDRS letters) & $+5.6 \pm 6.1$ & $+5.8 \pm 7.6$ & 0.785 \\
\hline Number of 10 -letter gainers, $\mathrm{n}(\%)$ & $6(26)$ & $9(33)$ & 0.758 \\
\hline Number of 15 -letter gainers, $\mathrm{n}(\%)$ & $3(13)$ & $4(15)$ & 1.000 \\
\hline Number of 10 -letter losers, $\mathrm{n}(\%)$ & $0(0)$ & $0(0)$ & - \\
\hline Number of 15 -letter losers, n (\%) & $0(0)$ & $0(0)$ & - \\
\hline Mean time to 10 -letter gain, mo & $3.6 \pm 1.9$ & $3.5 \pm 1.9$ & 0.880 \\
\hline
\end{tabular}

BCVA, best-corrected visual acuity; DDS, intravitreal dexamethasone delayed delivery system implant; ETDRS, Early Treatment Diabetic Retinopathy Study; IVB, intravitreal bevacizumab.

10- and 15- letter gainers and losers is graphically demonstrated in Figure 2. A trend was noted to suggest greater responsiveness to the dexamethasone implant compared with bevacizumab for eyes with greater number of previous anti-VEGF injections, but this was not statistically significant (Figure 3). A trend was also noted to suggest greater responsiveness to the dexamethasone implant compared with bevacizumab for eyes with worse baseline visual acuity, but this was not statistically significant (Figure 4).

The anatomical outcomes in the 2 groups are summarized and compared in Table 4. As noted, macular thickness and volume outcomes were all superior in the DDS group compared with the IVB group. The number (\%) of eyes considered "dry" on OCT as per study criterion of CST $<300 \mu \mathrm{m}$ was $1(4 \%), 0(0 \%)$, $0(0 \%), 0(0 \%), 2(9 \%), 0(0 \%)$, and $0(0 \%)$ in the IVB group and $10(37 \%), 11(42 \%), 9(35 \%), 11(41 \%), 13$ $(48 \%), 11(41 \%)$, and $11(41 \%)$ in the DDS group at monthly visits from Month 1 to Month 7. Central subfield thickness over time is shown in Figure 5, showing early reduction of macular thickness on OCT and a sustained deturgescence in the DDS group over the course of the study, with statistically significant difference in macular thickness at each visit compared with the IVB group. The macular volume showed a corresponding significant difference in outcomes at each visit (see Figure, Supplemental Digital Content 3, http://links. lww.com/A441, which graphically illustrates the total macular volume over time). Response to treatment as seen on OCT is illustrated in Figure 6, with the 2 eyes of the same subject enrolled in the 2 groups.

\section{Safety Measures}

No serious systemic safety concerns emerged during this study. The safety outcomes in the 2 groups are summarized and compared in Table 5. Regarding

Fig. 1. Change in visual acuity. The graph illustrates the change in visual acuity from baseline at each visit, comparing bevacizumab with the dexamethasone implant for persistent DME.

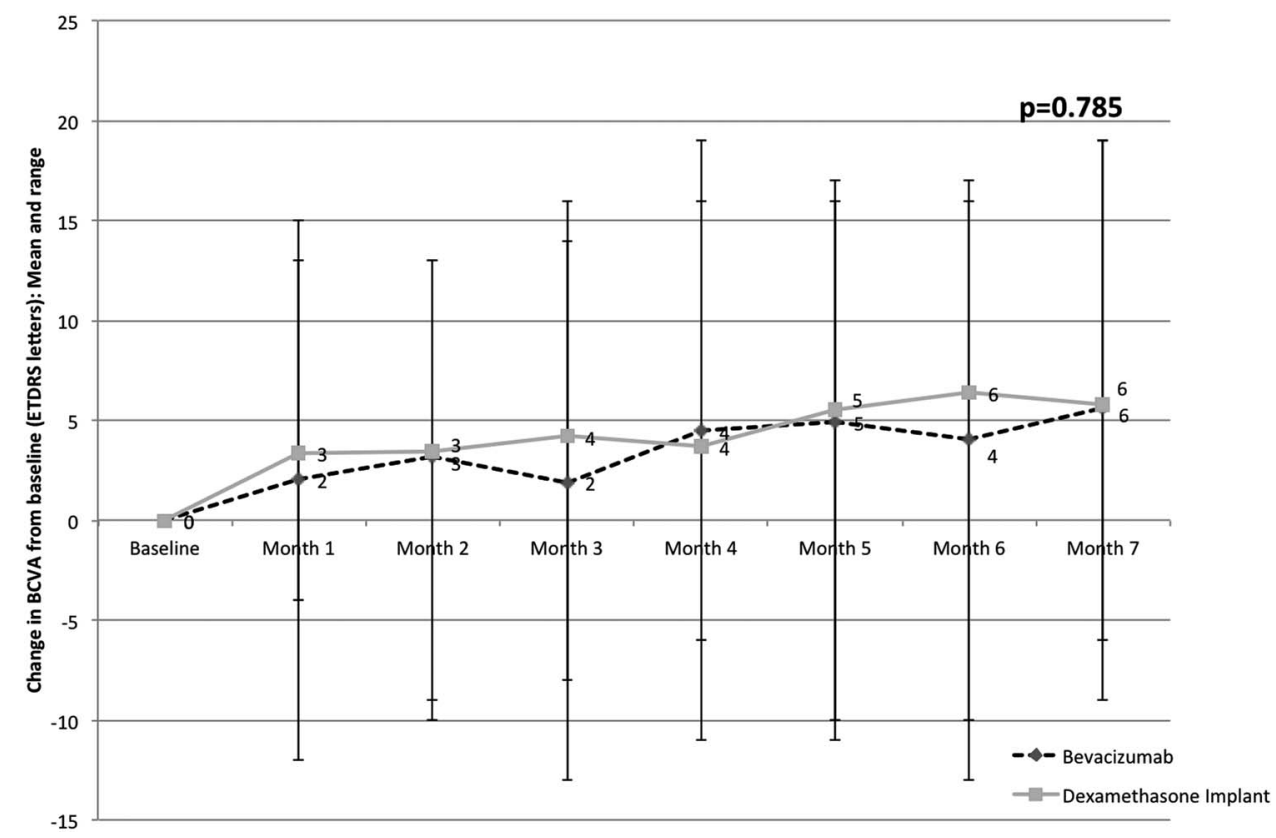




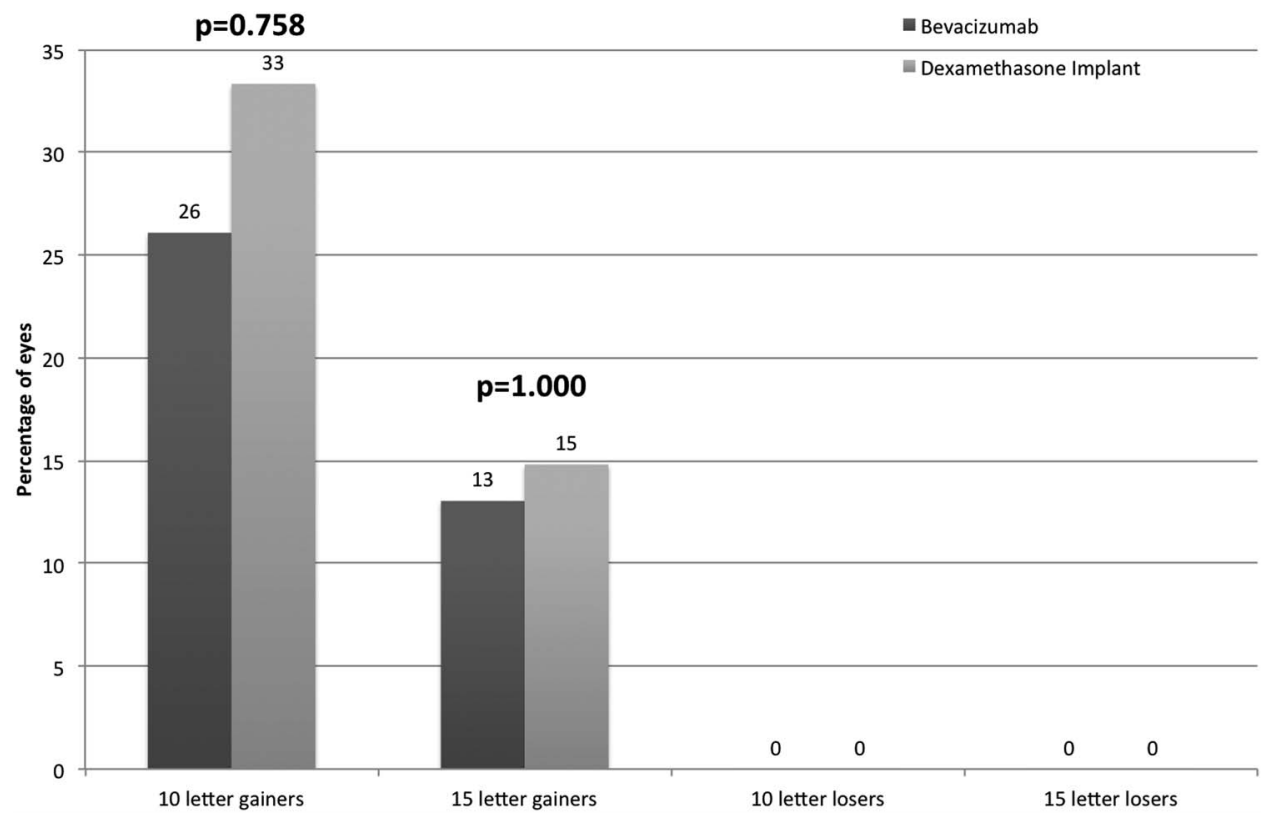

Fig. 2. Ten- and 15-letter gainers and losers. The graph illustrates the percentage of eyes with 10 and 15-letter gains, and 10- and 15-letter losses at Month 7, comparing bevacizumab with dexamethasone implant for persistent DME.

glaucoma-related outcomes, significantly greater proportion of patients in the DDS group had an IOP elevation and required glaucoma agents for IOP control; however, none of the eyes required laser or incisional surgery for glaucoma in either group. In the IVB group, none of the eyes had IOP $>21 \mathrm{mmHg}$ at any visit. In the DDS group, the number of eyes with IOP exceeding 21, 23, 25, and $30 \mathrm{mmHg}$ at each of the monthly visits is summarized in Table 6 . The absolute and the change in peripapillary retinal nerve fiber layer thickness were similar in both groups at the end of the study. The IOP trend over time in the 2 groups is shown in Figure 7. The number of ocular antihypertensive medications required for IOP control in the DDS group was significantly higher than that in the IVB group (see Figure, Supplemental Digital Content 4, http://links.lww.com/IAE/A442, which graphically illustrates the number of ocular antihypertensive required over time). Most eyes requiring ocular antihypertensive treatment were started on a dorzolamide/ timolol combination, whereas a few eyes were started on a single agent (brimonidine), as per physician discretion. Of those eyes that were already on either of those therapy, a new agent was required to be added in

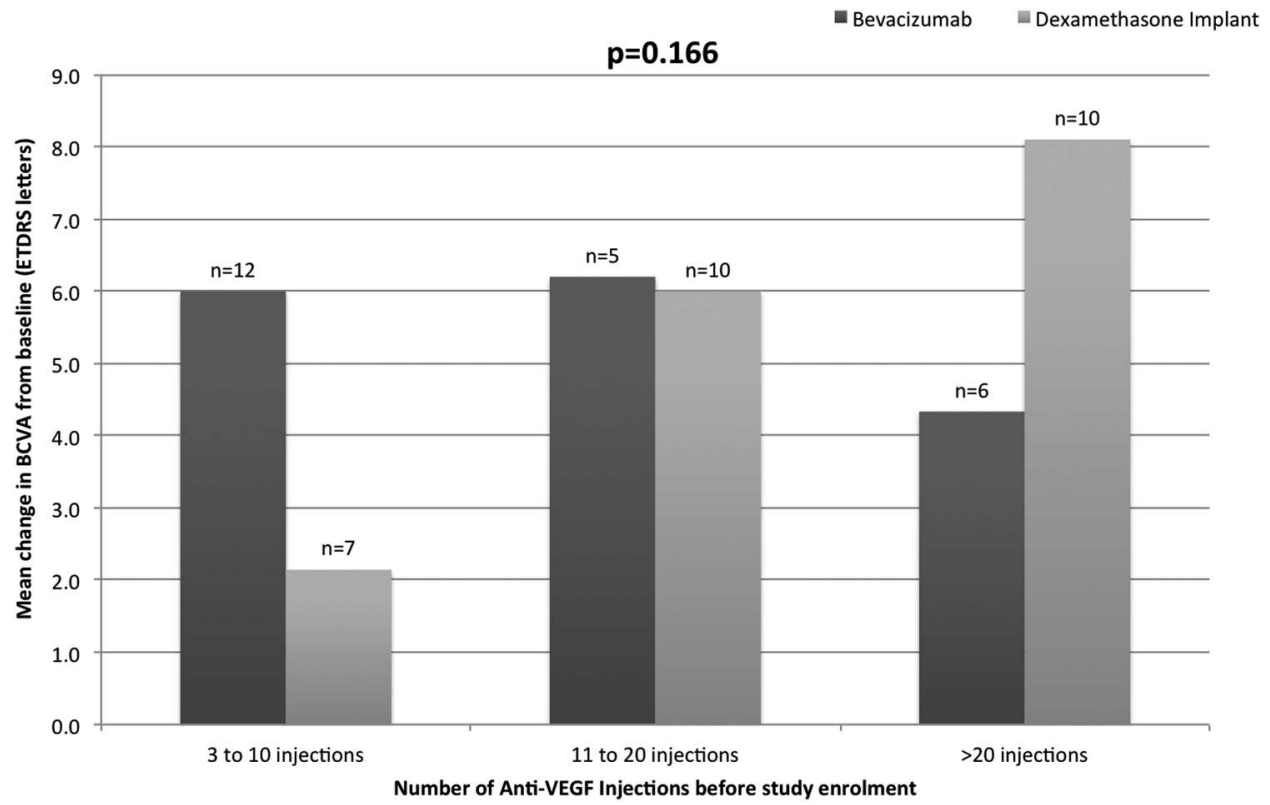

Fig. 3. Change in visual acuity and number of previous antiVEGF injections. The graph illustrates change in visual acuity from baseline to Month 7, based on the number of previous antiVEGF injections before study enrollment. It shows a nonsignificant trend toward greater visual acuity gain with dexamethasone in eyes with greater number of previous anti-VEGF injections. 
Fig. 4. Change in visual acuity and baseline visual acuity. The graph illustrates change in visual acuity from baseline to Month 7 , based on the baseline visual acuity. It shows a nonsignificant trend toward greater visual acuity gain with dexamethasone in eyes with poorer baseline visual acuity.

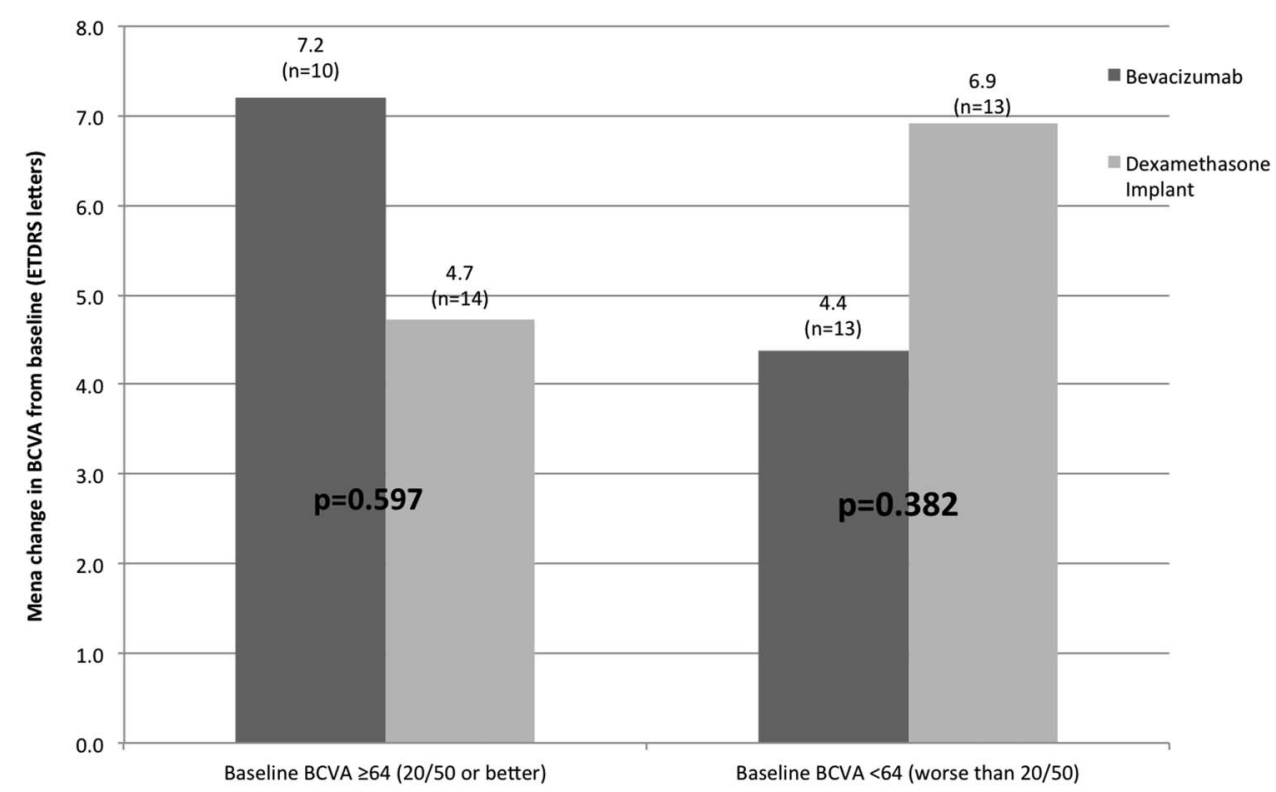

$0(0 \%)$ eyes in the IVB group, and $5(19 \%)$ eyes in the DDS group (2 eyes at Month 2, 2 eyes at Month 4, and one eye at Month 6). Cataract progression was noted in a higher proportion of DDS-group eyes, but this difference was not statistically significant during the short duration of the study. The specific ocular and systemic serious adverse events are summarized in Table 5, and occurred at a similar rate in both groups.

\section{Discussion}

While the pathophysiology of DME involves excess VEGF production as one of the key factors, it involves several other mechanisms as well. Anti-VEGF agents block and directly inhibit VEGF. Corticosteroid agents, however, have several mechanisms of action, including decrease in VEGF production and release, decrease in leukocyte migration, decrease in several proinflammatory cytokines and prostaglandins, downregulation of ICAM-1 expression, and also effect on retinal endothelial cells to enhance the barrier function of vascular tight junctions (antipermeability effect). ${ }^{13-16}$ With multiple targets of action, corticosteroids may theoretically offer better control in persistent DME.
Various corticosteroid agents that have been used for intravitreal use include triamcinolone acetonide, fluocinolone acetonide implants, and dexamethasone implants. DRCR protocol I showed that triamcinolone plus laser achieved similar visual gain as ranibizumab plus prompt or deferred laser groups in the pseudophakic subgroup at one year. ${ }^{3}$ The FAME study of intravitreal fluocinolone acetonide (Iluvien) in patients with refractory DME showed an improvement of at least 15 letters in $29 \%$ of patients receiving the $0.2 \mathrm{mg} /$ day insert; benefits sustained for over 3 years. ${ }^{17}$ Dexamethasone is 6 times more potent than triamcinolone acetonide but has a shorter half-life in vitreous. The dexamethasone implant (Ozurdex) is specifically designed for intraocular use with polylactic-co-glycolic acid copolymer packaging for prolonged and sustained release over several months. The MEAD study found that dexamethasone implants at both $0.7-\mathrm{mg}$ and 0.35 $\mathrm{mg}$ doses met the primary efficacy endpoint of significantly greater proportion of eyes with $>15$ letter best-corrected visual acuity improvement (22\% and $18 \%)$ at the end of 3 years compared with sham $(12 \%) .{ }^{18}$ The PLACID study showed that there were significantly greater 10 -line gainers (32\% vs. $17 \%)$

Table 4. Bevacizumab Versus Dexamethasone Implant for Persistent Diabetic Macular Edema: Anatomical Outcomes at Month 7

\begin{tabular}{lccr}
\hline \multicolumn{1}{c}{ Feature } & IVB Group, Mean $\pm \mathrm{SD}(\mathrm{n}=23$ Eyes $)$ & DDS Group, Mean \pm SD $(\mathrm{n}=27$ Eyes $)$ & $P$ \\
\hline Final CST, $\mu \mathrm{m}$ & $471 \pm 157$ & $336 \pm 89$ & 0.001 \\
Change in CST, $\mu \mathrm{m}$ & $-13 \pm 105$ & $-122 \pm 120$ & 0.001 \\
Final TMV, mm ${ }^{3}$ & $12.0 \pm 2.4$ & $10.7 \pm 1.4$ & 0.027 \\
Change in TMV, $\mathrm{mm}^{3}$ & $-0.5 \pm 2.8$ & $-1.0 \pm 1.4$ & 0.007 \\
\hline
\end{tabular}

DDS, intravitreal dexamethasone delayed delivery system implant; IVB, intravitreal bevacizumab; TMV, total macular volume. 


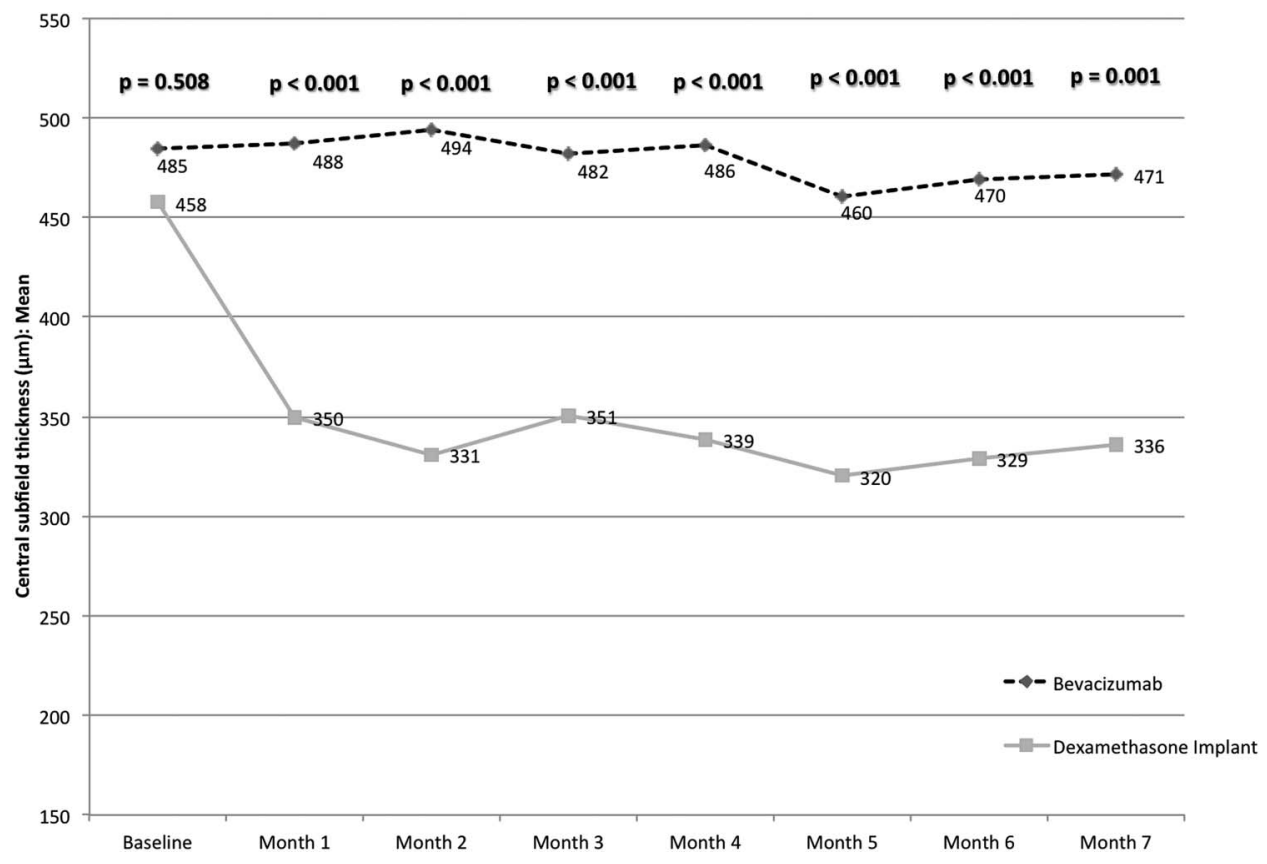

Fig. 5. Macular thickness. The graph illustrates the CST measured using OCT from baseline to Month 7, comparing bevacizumab with dexamethasone implant for persistent DME.

up to 9 months in the dexamethasone plus laser group compared with the sham plus laser group for DME, but the difference was not significant at 12 months. ${ }^{19}$

The duration of efficacy of dexamethasone was originally intended to be 6 months; however, it has been shown in several studies that, at least in persistent DME, its efficacy lasts approximately 3 months. ${ }^{12,20,21}$ Pacella et al demonstrated in their 6-month prospective study of eyes with persistent DME after multiple pre- vious therapies, including anti-VEGF agents, that the effect of a single Ozurdex injection has clinical efficacy lasting approximately 3 months to 4 months, with a statistically significant Early Treatment of Diabetic Retinopathy Study letter gain compared with baseline until Month 4 and a statistically significant CST reduction compared with baseline until Month 3. ${ }^{20}$ Totan et $\mathrm{al}^{21}$ conducted a similar 6-month prospective study and found the significant visual acuity gain lasting until Month 3 and a significant CST reduction lasting
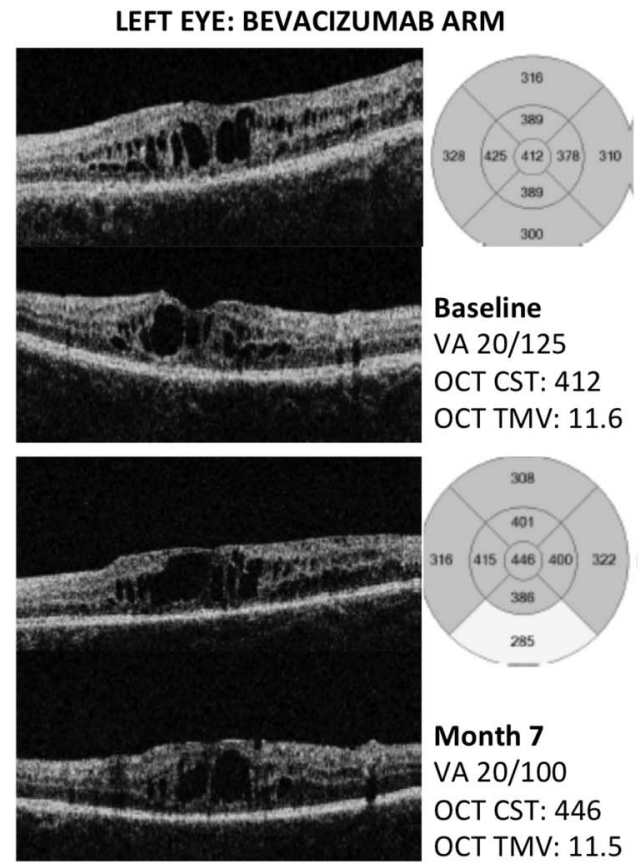
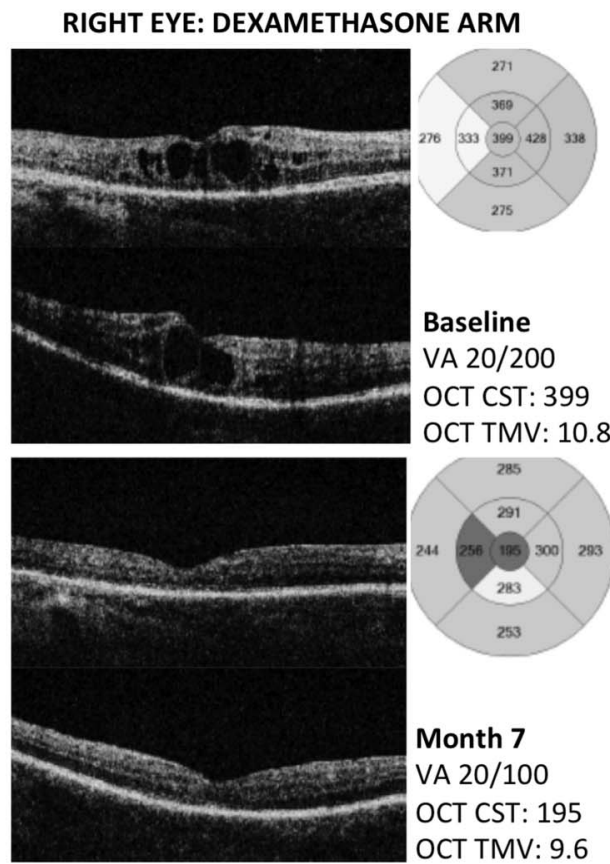

Month 7

VA 20/100

OCT CST: 195

OCT TMV: 9.6
Fig. 6. Macular OCT. Illustration of two eyes of the same patient with similar macular CST and total macular volume at baseline, with left eye randomized to bevacizumab and right eye to dexamethasone implant. At Month 7, the dexamethasone-arm eye had significantly better anatomical resolution of macular edema. Visual acuity gain is also specified. VA, visual acuity; TMV, Total macular volume. 
Table 5. Bevacizumab Versus Dexamethasone Implant for Persistent Diabetic Macular Edema: Safety Outcomes at Month 7

\begin{tabular}{|c|c|c|c|}
\hline Feature & $\begin{array}{l}\text { IVB Group, } \\
\text { Mean } \pm \text { SD } \\
(\mathrm{n}=23 \text { Eyes })\end{array}$ & $\begin{array}{l}\text { DDS Group, } \\
\text { Mean } \pm \text { SD } \\
(n=27 \text { Eyes })\end{array}$ & $P$ \\
\hline Final IOP, $\mathrm{mmHg}$ & $15 \pm 2$ & $17 \pm 3$ & 0.096 \\
\hline Change in IOP, $\mathrm{mmHg}$ & $-0.7 \pm 2.5$ & $+1.1 \pm 3.9$ & 0.049 \\
\hline Number of visits where IOP $>21 \mathrm{mmHg}$ & $0 \pm 0$ & $1.3 \pm 1.5$ & $<0.001$ \\
\hline $\begin{array}{l}\text { Number of visits at which ocular antihypertensive } \\
\text { medications were required to be used }\end{array}$ & $0.3 \pm 1.3$ & $2.4 \pm 2.8$ & 0.002 \\
\hline $\begin{array}{l}\text { Number of patients requiring addition of ocular } \\
\text { antihypertensive medications at any of the } \\
\text { visits during the study }\end{array}$ & $1(5)$ & $13(50)$ & 0.001 \\
\hline $\begin{array}{l}\text { Number of patients requiring laser or incisional } \\
\text { glaucoma surgery during the study }\end{array}$ & $0(0)$ & $0(0)$ & - \\
\hline $\mathrm{IOP}>21 \mathrm{mmHg}$ at least one visit, $\mathrm{n}(\%)$ & $0(0)$ & $14(52)$ & $<0.001$ \\
\hline Final RNFL, $\mu \mathrm{m}$ & $88 \pm 12$ & $91 \pm 11$ & 0.417 \\
\hline Change in RNFL, $\mu \mathrm{m}$ & $-1.2 \pm 5.2$ & $-3.3 \pm 6.5$ & 0.500 \\
\hline Cataract, n (\%) & $4(17)$ & $7(26)$ & 0.515 \\
\hline Endophthalmitis, n (\%) & $0(0)$ & $0(0)$ & - \\
\hline Ocular SAEs*, n (\%) & $3(13)$ & 2 (7) & 0.651 \\
\hline Systemic SAEst, $n(\%)$ & $4(17)$ & $1(4)$ & 0.167 \\
\hline
\end{tabular}

*Ocular SAEs included-IVB group: vitreous hemorrhage $(n=2)$ and cranial nerve VI palsy $(n=1)$; DDS group: vitreous hemorrhage $(n=1)$ and choroidal detachment $(n=1)$.

†Systemic SAEs included-IVB group: bradycardia $(n=1)$, pneumonia $(n=1)$, pyelonephritis $(n=1)$, and colon cancer $(n=1)$; DDS group: congestive heart failure $(n=1)$.

DDS, intravitreal dexamethasone delayed delivery system implant; IVB, intravitreal bevacizumab; RNFL, retinal nerve fiber layer; SAEs, serious adverse events.

until Month 6, after a single Ozurdex injection in persistent DME defined similarly. Our previous study comparing a combination of dexamethasone implants and bevacizumab with bevacizumab alone showed a similar period of efficacy of 3 months as well, with edema returning to baseline by Month $4 .^{12}$ In this study, a q3month dexamethasone implant was able to sustain the macular thickness control without any significant fluctuations over time (Figure 5).

Our present study has 2 additional findings - a trend toward greater visual acuity gain in eyes that have received a greater number of anti-VEGF injections previously and a trend toward greater visual acuity gain in eyes with poorer $(<20 / 50)$ baseline visual acuity. Although these trends are not statistically significant, they require further investigation because this study was not powered to test these differences.

It should be noted that cross-comparison between various studies evaluating persistent or refractory DME is challenging because of variations in definitions, enrollment criteria, duration of study, and types and frequency of treatments, among other factors. BEVORDEX was a randomized controlled trial that compared dexamethasone implants with bevacizumab in the treatment of center-involving DME unresponsive to macular laser and found that visual acuity gain was statistically similar $(+5.6$ vs. +8.9$)$, central macular thickness reduction was significantly greater $(-187$ vs. -122 ), and the number of injections required was significantly lower (2.7 vs. 8.6 over 12 months). ${ }^{22}$ However, more dexamethasone-treated eyes lost vision than bevacizumab-treated eyes, owing to the increased rate of cataract formation. ${ }^{22}$ The visual and anatomical outcomes of our study are in line with the BEVORDEX study, and the rate of cataract formation in our study was not significantly different between the two groups, most likely owing to the shorter follow-up.

An important consideration against using intravitreal corticosteroid agents includes their potential for IOP elevation and glaucoma. One of the

Table 6. Number of Eyes With IOP Elevation at Monthly Visits in the Dexamethasone Implant Group

\begin{tabular}{lccccccc}
\hline IOP, mmHg & $\begin{array}{c}\text { Month 1, } \\
\mathrm{n}(\%)\end{array}$ & $\begin{array}{c}\text { Month 2, } \\
\mathrm{n}(\%)\end{array}$ & $\begin{array}{c}\text { Month 3, } \\
\mathrm{n}(\%)\end{array}$ & $\begin{array}{c}\text { Month 4, } \\
\mathrm{n}(\%)\end{array}$ & $\begin{array}{c}\text { Month 5, } \\
\mathrm{n}(\%)\end{array}$ & $\begin{array}{c}\text { Month 6, } \\
\mathrm{n}(\%)\end{array}$ & $\begin{array}{c}\text { Month 7, } \\
\mathrm{n}(\%)\end{array}$ \\
\hline$>21$ & $3(11)$ & $10(37)$ & $2(7)$ & $3(11)$ & $8(30)$ & $5(19)$ & $2(7)$ \\
$>23$ & $3(11)$ & $5(19)$ & $0(0)$ & $3(11)$ & $4(15)$ & $3(11)$ & $1(4)$ \\
$>25$ & $3(11)$ & $3(11)$ & $0(0)$ & $1(4)$ & $1(4)$ & $1(4)$ & $1(4)$ \\
$>30$ & $1(4)$ & $1(4)$ & $0(0)$ & $1(4)$ & $1(4)$ & $0(0)$ & $0(0)$ \\
\hline
\end{tabular}




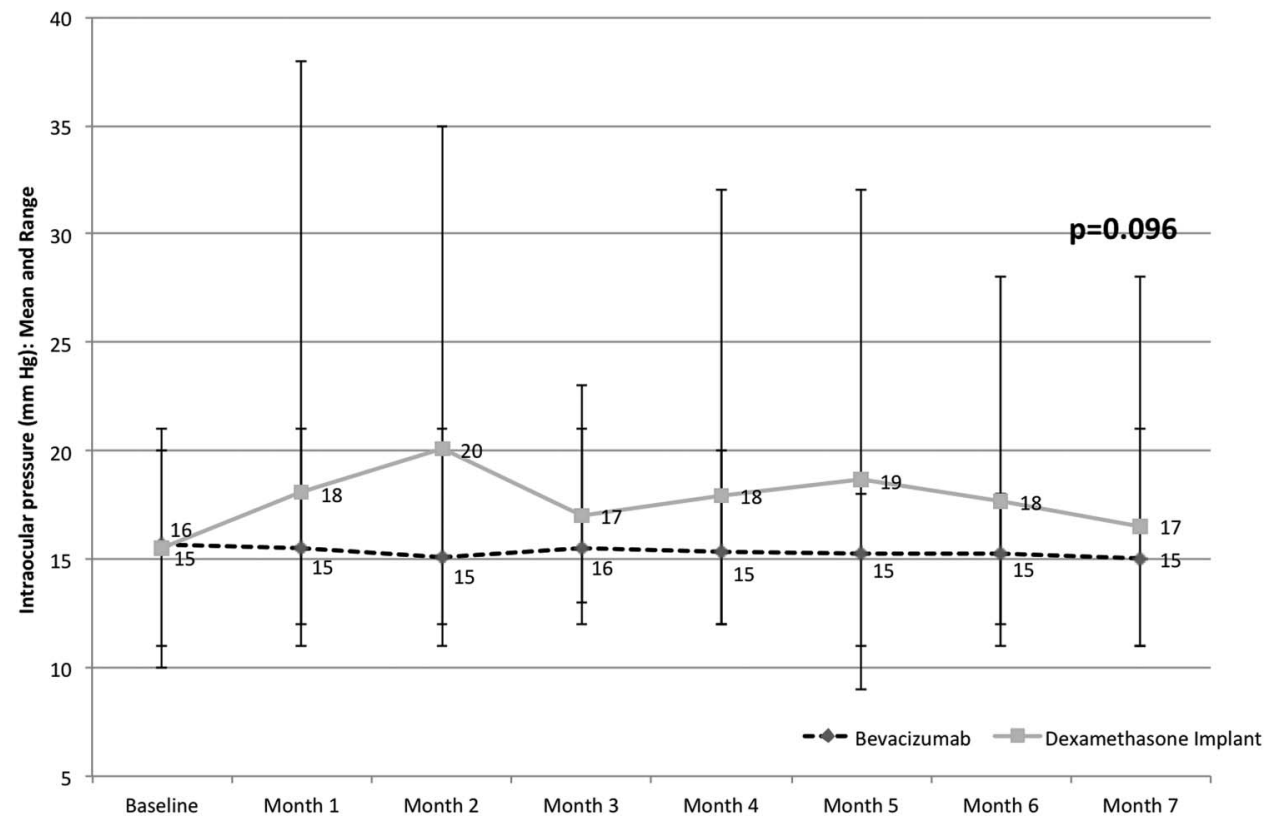

Fig. 7. Intraocular pressure. The graph illustrates the IOP from baseline to Month 7, comparing bevacizumab with dexamethasone implant for persistent DME. strengths of our study is specific analyses of glaucoma-related outcomes. Intraocular pressure elevation occurred in a significantly greater number of eyes in the dexamethasone arm and a correspondingly greater number of ocular antihypertensive agents were required in this group. However, optic nerve OCT failed to show any significant difference in absolute or relative change in retinal nerve fiber layer thickness at Month 7. Also, none of the eyes required laser or surgical glaucoma treatments. It is noteworthy that although the dexamethasoneimplant group patients required significantly fewer injections than the bevacizumab group, they still needed follow-up every 4 weeks to 6 weeks for IOP monitoring and management, thereby not translating into fewer patient visits. Second, although a detailed cost-effectiveness analysis is beyond the scope of our study, another consideration against dexamethasone implant use is its significantly higher cost compared with bevacizumab, despite fewer injections. However, dexamethasone implants would be less expensive than using either ranibizumab (Lucentis, Genentech, Inc., South San Fancisco, CA) or aflibercept (Eylea, Regeneron Pharmaceuticals, Inc., Tarrytown, NY), per label.

Some of the limitations of our study include the short duration of follow-up and the small sample size. Future studies on persistent DME with longer followup and detailed analysis of glaucoma-related outcomes would be helpful. In addition, the effect of switching over to bevacizumab after an initial good response to dexamethasone for patients with significant IOP elevation/steroid response would be helpful as well.
Moreover, our study suggests a trend indicating that earlier treatment of chronic macular edema may result in a better final visual acuity. Thus, the use of alternative treatments to anti-VEGF agents alone should likely be considered earlier in the treatment process. Finally, the trends suggested regarding the merits of dexamethasone, especially with early treatment, is worthy of further analyses.

Key words: bevacizumab, dexamethasone, diabetic macular edema, optical coherence tomography, sustained release implant, vascular endothelial growth factor.

\section{References}

1. CDC. Centers for Disease Control and Prevention-About Vision Health-Common Eye Disorders-Vision Health Initiative (VHI). http://www.cdc.gov/visionhealth/basic_information/ eye_disorders.htm. Accessed on August 16, 2015.

2. Varma R, Bressler NM, Doan QV, et al. Prevalence of and risk factors for diabetic macular edema in the United States. JAMA Ophthalmol 2014;132:1334-1340.

3. Elman MJ, Aiello LP, Beck RW, et al. Randomized trial evaluating ranibizumab plus prompt or deferred laser or triamcinolone plus prompt laser for diabetic macular edema. Ophthalmology 2010;117:1064-1077.e35.

4. Elman MJ, Bressler NM, Qin H, et al. Expanded 2-year follow-up of ranibizumab plus prompt or deferred laser or triamcinolone plus prompt laser for diabetic macular edema. Ophthalmology 2011;118:609-614.

5. Nguyen QD, Brown DM, Marcus DM, et al. Ranibizumab for diabetic macular edema: results from 2 phase iii randomized trials: RISE and RIDE. Ophthalmology 2012;119: 789-801.

6. Do DV, Nguyen QD, Boyer D, et al. One-year outcomes of the da VINCI study of VEGF trap-eye in eyes with diabetic macular edema. Ophthalmology 2012;119:1658-1665. 
7. Diabetic Retinopathy Clinical Research Network, Wells JA, Glassman AR, Ayala AR, et al. Aflibercept, bevacizumab, or ranibizumab for diabetic macular edema. N Engl J Med 2015; 372:1193-1203.

8. Brown DM, Nguyen QD, Marcus DM, et al. Long-term outcomes of ranibizumab therapy for diabetic macular edema: the 36-month results from two phase III trials: RISE and RIDE. Ophthalmology 2013;120:2013-2022.

9. Dhoot DS, Pieramici DJ, Nasir M, et al. Residual edema evaluation with ranibizumab $0.5 \mathrm{mg}$ and $2.0 \mathrm{mg}$ formulations for diabetic macular edema (REEF study). Eye (Lond) 2015;29: 534-541.

10. Elman MJ, Ayala A, Bressler NM, et al. Intravitreal ranibizumab for diabetic macular edema with prompt versus deferred laser treatment: 5-year randomized trial results. Ophthalmology 2015;122:375-381.

11. Elman MJ, Qin H, Aiello LP, et al. Intravitreal ranibizumab for diabetic macular edema with prompt versus deferred laser treatment. Ophthalmology 2012;119:2312-2318.

12. Maturi RK, Bleau L, Saunders J, et al. A 12-month, singlemasked, randomized controlled study of eyes with persistent diabetic macular edema after multiple anti-vegf injections to assess the efficacy of the dexamethasone-delayed delivery system as an adjunct to bevacizumab compared with contin. Retina 2015;35:1604-1614

13. Miyamoto K, Khosrof S, Bursell SE, et al. Prevention of leukostasis and vascular leakage in streptozotocin-induced diabetic retinopathy via intercellular adhesion molecule-1 inhibition. Proc Natl Acad Sci U S A 1999;96:10836-10841.

14. Felinski EA, Antonetti DA. Glucocorticoid regulation of endothelial cell tight junction gene expression: novel treatments for diabetic retinopathy. Curr Eye Res 2005;30:949-957.
15. Sohn HJ, Han DH, Kim IT, et al. Changes in aqueous concentrations of various cytokines after intravitreal triamcinolone versus bevacizumab for diabetic macular edema. Am J Ophthalmol 2011;152:686-694.

16. Wilson CA, Berkowitz BA, Sato Y, et al. Treatment with intravitreal steroid reduces blood-retinal barrier breakdown due to retinal photocoagulation. Arch Ophthalmol 1992; 110:1155-1159.

17. Campochiaro PA, Brown DM, Pearson A, et al. Sustained delivery fluocinolone acetonide vitreous inserts provide benefit for at least 3 years in patients with diabetic macular edema. Ophthalmology 2012;119:2125-2132.

18. Boyer DS, Yoon YH, Belfort R, et al. Three-year, randomized, sham-controlled trial of dexamethasone intravitreal implant in patients with diabetic macular edema. Ophthalmology 2014;121: 1904-1914.

19. Callanan DG, Gupta S, Boyer DS, et al. Dexamethasone intravitreal implant in combination with laser photocoagulation for the treatment of diffuse diabetic macular edema. Ophthalmology 2013;120:1843-1851.

20. Pacella E, Vestri AR, Muscella R, et al. Preliminary results of an intravitreal dexamethasone implant (Ozurdex) in patients with persistent diabetic macular edema. Clin Ophthalmol 2013;7:1423-1428.

21. Totan Y, Güler E, Gürağaç FB. Dexamethasone intravitreal implant for chronic diabetic macular edema resistant to intravitreal bevacizumab treatment. Curr Eye Res 2016;41: 107-113.

22. Gillies MC, Lim LL, Campain A, et al. A randomized clinical trial of intravitreal bevacizumab versus intravitreal dexamethasone for diabetic macular edema: the BEVORDEX study. Ophthalmology 2014;121:2473-2481. 